
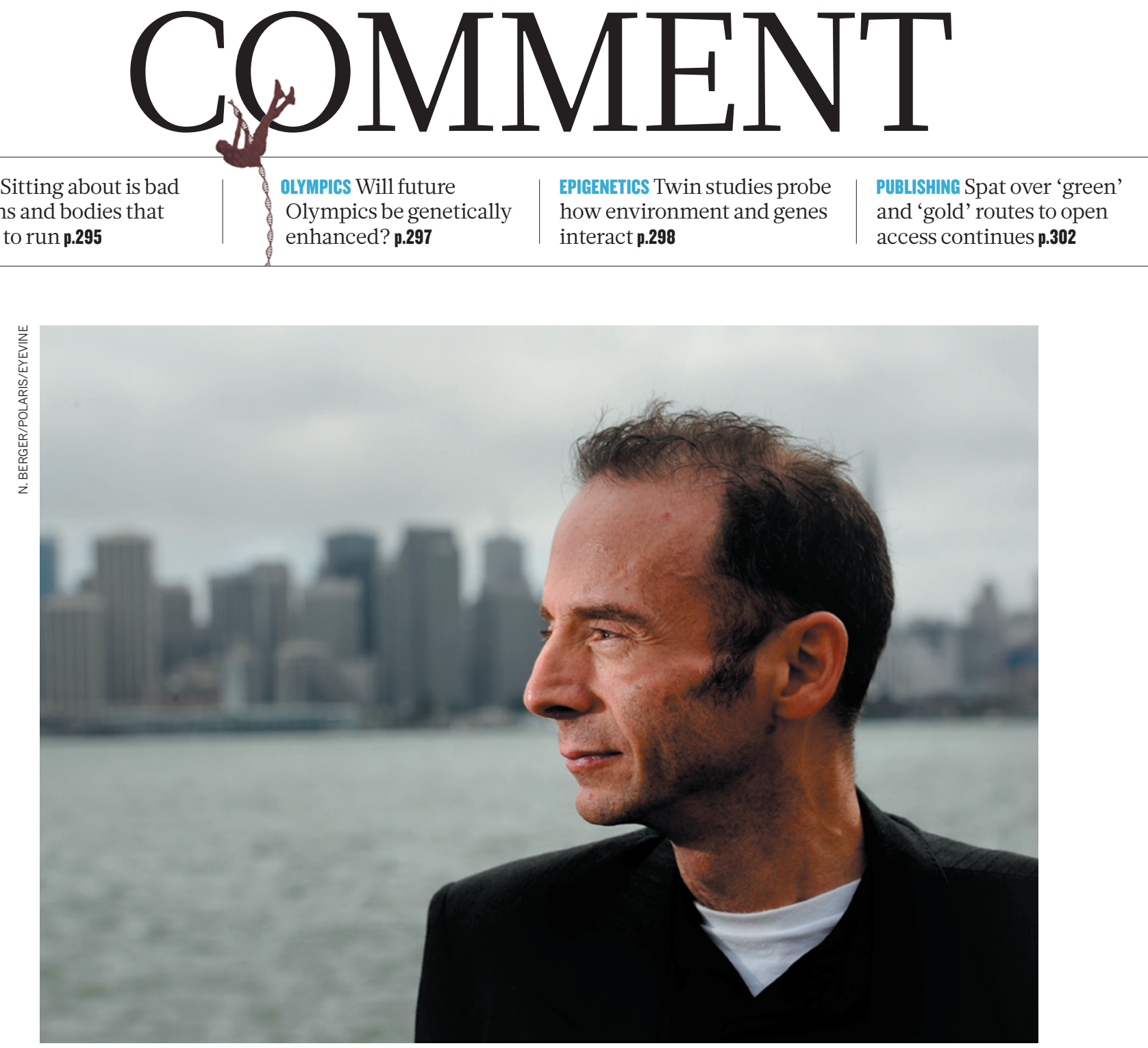

The 'Berlin Patient,' Timothy Brown, has been cured of HIV since 2007. His story has renewed interest in cure research.

\title{
Towards a cure for HIV
}

\author{
Steven G. Deeks and Françoise Barré-Sinoussi present \\ an international research agenda to seek out a cure for AIDS.
}

$\mathrm{O}$ ne of the greatest achievements of modern medicine has been the development of combination antiretroviral (ARV) therapy for HIV. Today, fewer than half of the world's people who need treatment have access to therapy. A substantial and sustained increase in funding will be required to effectively treat the global population (see 'Cost of managing HIV'). And this life-saving therapy has limitations - medicines have side effects and must be taken daily, and HIV can develop resistance. Clearly, a new approach to tackling HIV is needed.

In 2007, an HIV-infected man in Berlin received a transplant of haematopoietic stem cells from a naturally HIV-resistant donor, and then he stopped HIV therapy ${ }^{1}$. He has now been free of readily detectable virus in the absence of therapy for more than five years. In other words, he is cured. His experience suggests that HIV infection might one day be curable.

One of the key priorities of the International AIDS Society (IAS) is to promote and facilitate the search for a safe, affordable and scalable cure. The multidisciplinary IAS Scientific Working Group on HIV Cure

\section{DNATURE.COM} Read more about the HIV/AIDS pandemic:

go.nature.com/xey8dc has developed a broad and ambitious set of priorities for cure research (see 'Priorities for HIV cure research'). Some of these research questions have been pursued for decades, but the focus has been mainly on improving therapy or developing vaccines. Unique perspectives on these old questions will almost certainly be needed for cure research to succeed.

Cure research is not completely new. Several high-profile approaches were attempted soon after the development of combination therapy ${ }^{2}$. But these attempts failed, and the field shifted towards optimizing therapy so that it could be taken indefinitely. Since 
then, only a few scientists have continued to do cure research, working without a clear source of funding and despite a widespread sense that a cure is not possible. Increasing the number of scientists involved in this effort will be a crucial first step.

ARV therapy cannot cure HIV mainly because the virus is able to integrate its DNA into the genomes of long-lived immune cells called memory CD4 T cells, preventing the immune system from recognizing and clearing these cells. Determining how this 'latency' works, where infected cells reside and how latent infection could be reversed are the focal points of most ongoing cure research. Such work is based on the assumption that if all infected memory CD4 T cells could be identified and forced to make virus while ARVs inhibit that virus from infecting new cells, then the host immune system could identify and kill all infected cells, thereby achieving a cure. But it is unclear whether the immune system has the capacity to kill infected cells, even if they could be forced to start making virus. It is also unclear whether other cell types harbour HIV during long-term therapy, or whether ARV s completely inhibit the virus.

It may not be necessary to completely eradicate the virus in the individual, however. In about $1 \%$ of people infected with HIV, the virus is naturally controlled, such that their risks of disease progression and transmission are minimal. Scientists have studied these 'elite controllers' in search of a path towards developing a vaccine. Elite controllers could also provide clues about how to control, if not eliminate, established infection.

Funding remains a problem. The US National Institutes of Health (NIH) devoted about US\$56 million to cure-related work in 2011, although it plans to invest more in the future. The French National Agency for Research on AIDS and Viral Hepatitis provided more than $€ 7$ million (US\$8.6 million) last year. Since 2006, the statefunded California Institute of Regenerative

\section{DEVISING A CURE}

\section{Priorities for HIV cure research}

- Determine the mechanisms that maintain HIV persistence. This includes defining the role of latency, replication and cell proliferation. - Determine the tissue and cellular sources of persistent HIV infection in animal models and in people who undergo long-term antiretroviral (ARV) treatment.

- Determine the origins of immune activation and inflammation in the presence of ARVs and their consequences for viral persistence. - Determine host and immune mechanisms that control infection but allow viral persistence.

- Develop and validate assays to measure persistent HIV infection. - Develop and test therapeutic strategies to safely eliminate latent infection, including reversal of latency and clearing of latently infected cells. - Develop and test strategies to enhance the host response's capacity to control active viral replication.

Medicine has spent more than $\$ 40$ million on gene-therapy approaches that would, for example, make cells resistant to HIV infection. Foundations have also been supportive. The Foundation for AIDS Research, based in New York, contributed \$4.1 million to the effort in 2011. Still, although a price tag for the IAS research priorities cannot be calculated, it is clear that more resources - perhaps hundreds of millions of dollars per year will be needed to find a cure.

This extra funding should not be diverted from other high-priority research areas such as vaccine development, nor from life-saving

\section{COST OF MANAGING HIV}

Researchers have proposed a plan (investment framework) ${ }^{3}$ that would increase global access to HIV therapy and decrease the number of new infections in low- and middle-income countries over the coming decade. For the plan to succeed, however, a large increase over current (baseline) spending is needed.

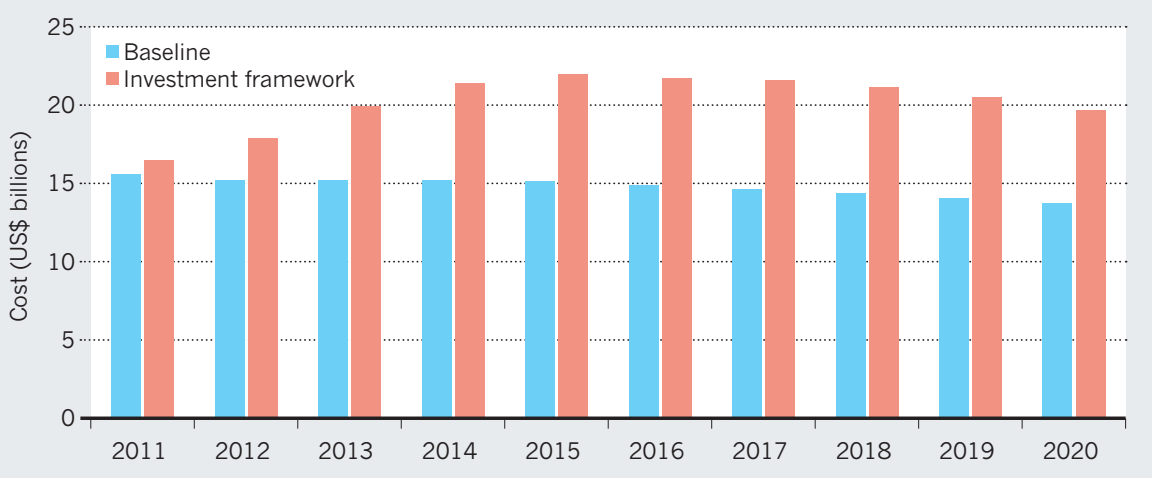

and underfunded treatment programmes. The push should come from new funding sources, such as private foundations or governments that are fighting growing epidemics (such as China, India and Brazil).

It is also not enough to develop and fund a research agenda. The research infrastructure must be improved. The community must establish large, multinational and multidisciplinary collaborations specifically for cure research. The NIH Martin Delaney Collaboratory grants ( $\$ 14$ million a year for up to five years), which are focused on collaborative efforts to this end, are a first step.

Scientists also need more support for HIV research in animals. Although they can infect macaques with the simian version of HIV, many scientists cannot afford to treat animals with ARV therapy over long periods to replicate the physiology of a human, as it costs tens of thousands of dollars per year per animal. Resources will need to be made available for the development, validation and optimization of such non-human primate models, and for the development of small-animal models for more high-throughput studies.

The research community must also address the ethical issues that arise in HIV cure research, in which scientists must test new and potentially very toxic drugs in individuals who are receiving long-term ARV therapy. By definition, these people have access to therapy and are doing well, so the potential risks to them will need to weighed against the potential benefits for the larger community. Strong community support is needed to ensure that patients and their care-givers are fully engaged and informed about the risks and benefits of curative studies.

The barriers to curing HIV are real, and they may prove to be insurmountable. Scientists who have in the past spoken about the promise of a cure have experienced backlash when the cure did not materialize. It is the responsibility of organizations such as the IAS to encourage and enable research in this area, but to do so responsibly, without hype or false promises.

Steven G. Deeks is a professor of medicine at the University of California, San Francisco, California 94110, USA. Françoise Barré-Sinoussi is a professor of virology at the Institut Pasteur, Institut National de la Santé et de la Recherche Médicale, Paris 75724, France. She is also IAS president-elect.

e-mail:sdeeks@php.ucsf.edu

1. Hütter, G. et al. N. Engl. J. Med. 360, 692-698 (2009).

2. The International AIDS Society Scientific Working Group on HIV Cure. Nature Rev. Immunol. (in the press).

3. Schwartländer, B. et al. Lancet 377, 2031-2041 (2011).

S.G.D. declares competing financial interests; see go.nature.com/bjntyh. 